Proceedings

\title{
Embedded Wearable Integrating Real-Time Processing of Electromyography Signals ${ }^{+}$
}

\author{
Paolo Gentile 1,2, Marco Pessione ${ }^{2}$, Antonio Suppa ${ }^{3,4}$, Alessandro Zampogna ${ }^{3}$ \\ and Fernanda Irrera ${ }^{1, *}$ \\ 1 Department of Information Engineering, Electronics and Telecommunications, \\ Sapienza University of Rome (I), Rome, Italy; p.gentile7@gmail.com \\ 2 STMicroelectronics, Agrate (I), Italy; marco.pessione@st.com \\ 3 Department of Neurology and Psychiatry, Sapienza University of Rome (I), Rome, Italy; \\ antonio.suppa@uniroma1.it (A.S.); alessandro.zampogna@gmail.com (A.Z.) \\ 4 IRCSS-NEUROMED, Pozzilli, IS (I), Italy \\ * Correspondence: fernanda.irrera@uniroma1.it; Tel.: +39-06-44585440 \\ + Presented at the Eurosensors 2017 Conference, Paris, France, 3-6 September 2017. \\ Published: 9 August 2017
}

\begin{abstract}
We realized a non-invasive wearable device able to record muscle activity using patch electrodes positioned on the skin over the muscle. It is an integrated system-on-board developed for the detection of several physical and physiologic human parameters which includes specific circuits for detecting the surface electromyography signal and algorithms for the real-time data processing optimized to low computational load. In real time, the proposed system dissipates only $26 \mathrm{~mW}$ and guarantees $20 \mathrm{~h}$ battery autonomy. The system exhibits performance comparable with those achieved with state-of-art wired equipment used in the hospitals, but with the advantage of being an embedded wearable wireless device.
\end{abstract}

Keywords: wearable for healthcare; embedded system; surface electromyography

\section{Introduction}

Application of ICT and sensor technologies to healthcare is an innovative strategy to treat the diseases and help doctors in managing specific diseases in all those situations in which the conventional methods of medicine is poorly effective. In this frame, wearables devices and body sensor networks are attracting most attention for the treatment of chronic diseases of elderly, who need monitoring of the symptoms and adjustment of pharmacological therapies [1,2].

The surface electromyography (sEMG) is a diagnostic technique that detects in real time the muscle activity using patch electrodes properly positioned on the skin over the studied muscle. This technique is not invasive, as opposed to the fine wire EMG, and is more comfortable and tolerated by patients. It is extremely useful in those cases in which it is necessary to monitor for (relatively) long time the muscle activity and to detect and distinguish voluntary contractions of muscles. This is the case of neurodegenerative diseases involving motor disorders. Cramps, muscle contractions, abnormalities in neuromuscular functions and a variety of symptoms related to the progression of neurodegenerative diseases can be studied objectively and quantitatively by sEMG. Currently, sEMG is performed by means of laboratory wired equipment. This poses a number of problems. First of all, the test needs to be performed during outpatient visits and therefore it can last a few minutes at most; second, the presence of wires implies space limitations in the sense that the patient cannot walk or make any free movement; third, wires introduce movement artifacts and last but not the least, noise of the power supply at $50 \mathrm{~Hz}$, which lies exactly in the most meaningful portion of the spectrum. 
To overcome these problems, we realized a wireless wearable embedded system able to record sEMG signal for long time, perform the signal processing, save data on a microSD (monitoring operation) or transmit them to a PC via Bluetooth Low Energy.

\section{The System}

Hardware. To get reliable clinical and physiological information from neuromuscular functionality, the sEMG needs to detect the exact timing and duration of the muscle activities. The typical raw sEMG signal is sketched in Figure 1. Regardless of the type of physical connection used (fine wires or patch electrodes), the frequency components are typically in the band between 3 and $500 \mathrm{~Hz}$ with a main content between 10 and $250 \mathrm{~Hz}$. In the case of the sEMG amplitude is typically between 0.1 and $2.0 \mathrm{mV}$ (which rises up to $5.0 \mathrm{mV}$ in very special cases as for athletes) [3].

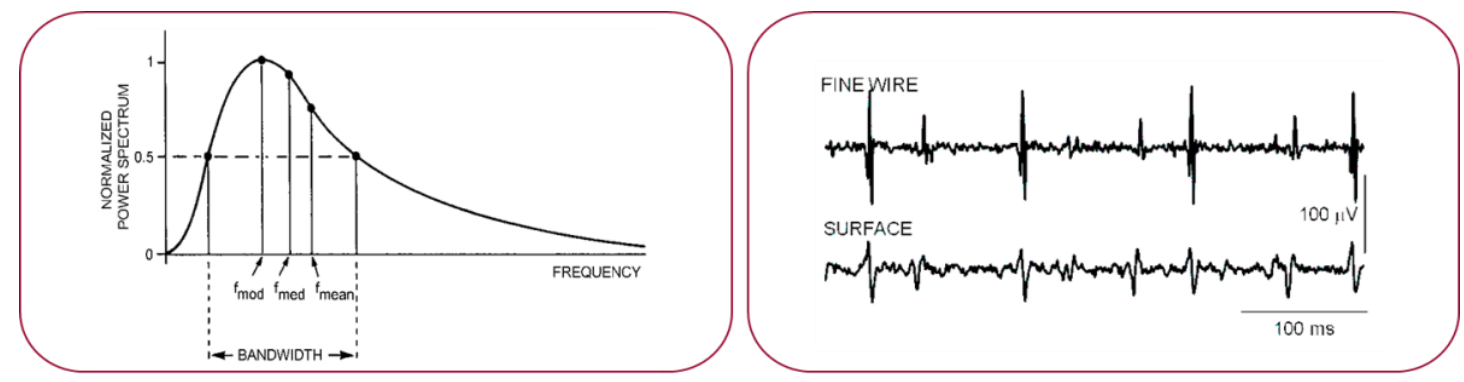

Figure 1. Sketch of a typical raw EMG signal recorded with fine wires or patch electrodes.

The starting point of the hard device was an integrated system-on-board (Bio2Bit Move) developed for the detection of several physical and physiologic human parameters (shown in Figure 2). The Bio2Bit Move contains an ultra-low power active front end for the acquisition of EMG signal, an ultra-low power MP ARM Cortex M4 at 32 bit, a Bluetooth Low Energy, a $592 \mathrm{mWh}$ battery, a micro-USB connector. In general, the blocks of a circuit for sEMG signal acquisition are displayed in Figure 2c.

The distance between the electrodes is equal to $20 \mathrm{~mm}$ according to international consensus of the SENIAM (a European concerted action in the EU Biomedical Health and Research program). The electrodes clips are integrated into the device package so that the distance remains fixed (as shown in Figure 2a). This avoids artifacts in the sEMG signal due to wire movement.
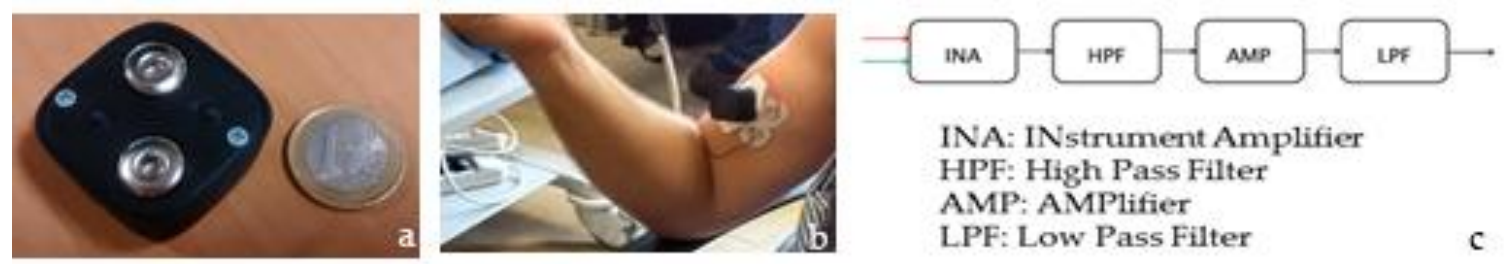

Figure 2. Sketch of the Bio2Bit Move: (a) the electrodes; (b) case of use; (c) blocks for sEMG signal acquisition.

Software. The choice of algorithms can be brought back to two main categories depending on whether or not it is required that the processed signal is known a priori. To be more general, we decided to implement an algorithm independent of the a priori knowledge, because it gives greater flexibility and is not related to any specific muscle. In fact, in the case of voluntary muscle contractions, the sEMG signal is stochastic and denotes a sudden variation of both amplitude and frequency, deriving from the activation of the action potential. Therefore, we focused onto the TeagerKaiser Energy Operator (TKEO), which puts in evidence the instantaneous increase of the action potential and reduces the baseline noise [4]. A threshold algorithm was then implemented in TKEO's domain for detecting muscle activity, taking into consideration the minimum period of muscular activity, the minimum period of muscle inactivity and the margin of accuracy in the estimation of 
such measures [5,6]. An example of TKEO normalization is shown in Figure 3. The signal processing chain is shown in Figure 4. Signal processing of the sEMG signal was based on the FIR DC and HP filtering, the TKEO normalization and the RMS smoothing.

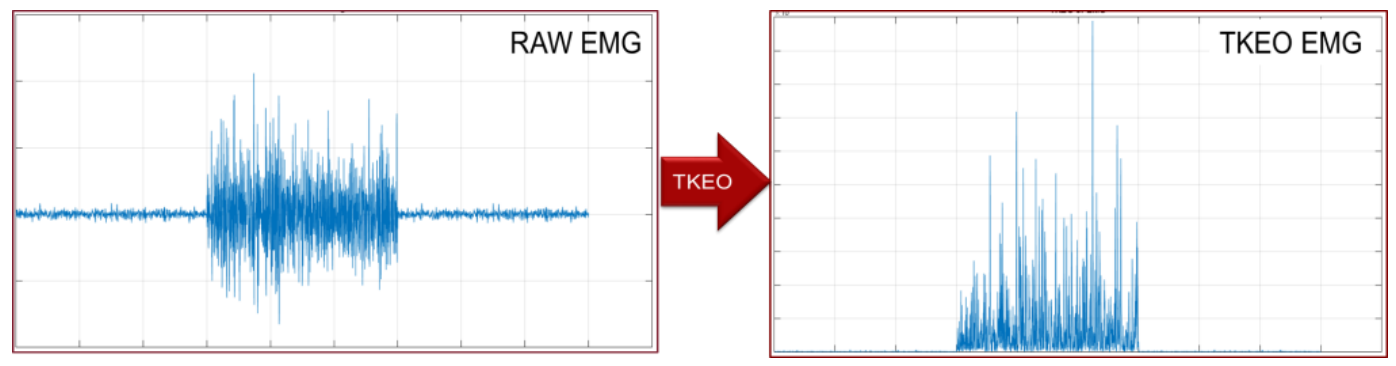

Figure 3. Raw and TKEO EMG signal, on the same time and amplitude scale.

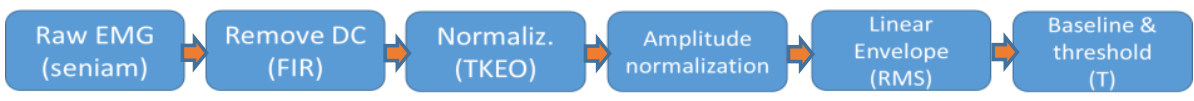

Figure 4. Data processing chain.

For the determination of the threshold $(\mathrm{T})$, the period of muscular inactivity before a contraction was studied. Then, we defined: $T=$ mean $(E M G(t w))+j \cdot \operatorname{std}(E M G(t w))$, to distinguish the muscle activity time periods from inactivity ones. In that equation, mean is the average value, std the standard deviation, $j$ a dynamic factor, $t w$ the time window (in this case is of $500 \mathrm{~ms}$ ). Figure $5 \mathrm{a}$ shows the detection of the muscle activity with RMS (blue) and dynamic threshold factor $j$ (green) in $t w=200$ ms. An example of the processed sEMG signal is displayed in Figure $5 b$ in the same $t w$.
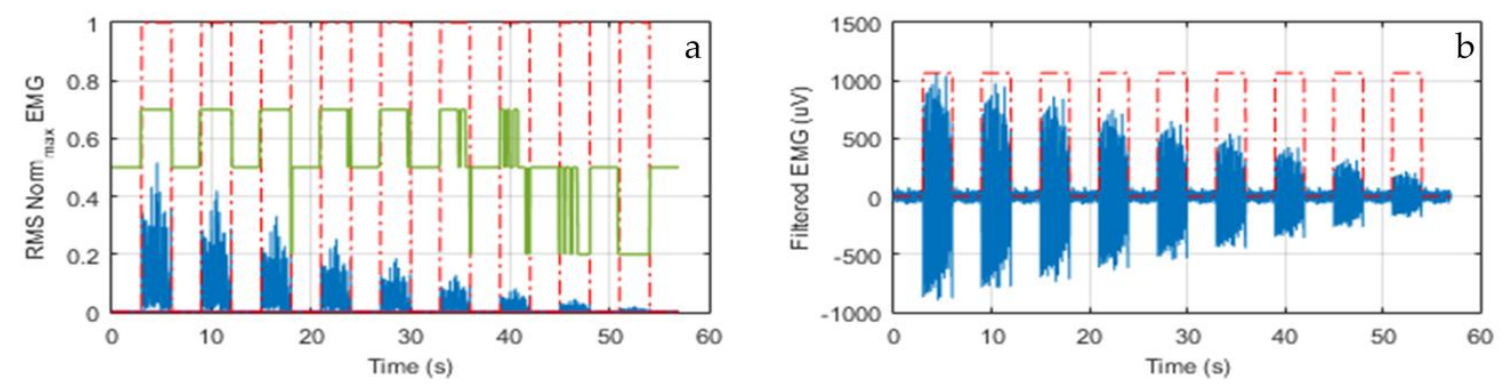

Figure 5. (a) The RMS signal (blue) and factor $j$ (green); (b) An example of the EMG signal in the same tw.

Tests and Performances. Systematic tests were performed recording contemporarily the sEMG signal with our wearable device and a state-of-art commercial wired equipment (Digitimer D360, Digitimer Ltd., Welwyn Garden City, Hertfordshire, UK), as shown, for example, in Figure 2b. First of all, we noticed that the Digitimer exhibited a much worse baseline due to the electrical grid noise (especially for the muscles of small dimension, as the abductor pollicis brevis), as shown in Figure 6a, where a zoom of the two synchronized trace baselines is displayed. However, if the Digitimer Notch filter at $50 \mathrm{~Hz}$ was activated, the information around $50 \mathrm{~Hz}$ was lost and this is very penalizing since $50 \mathrm{~Hz}$ lies in the mid of the meaningful spectrum. This is shown in Figure 6, where the FFT of signals are displayed. As a result, our wearable device showed excellent performance in terms of sensitivity and specificity in muscle activity detection and timing. In fact, the traces obtained with our wearable device were always perfectly superimposed on the time scale with those obtained with the Digitimer D360, as demonstrated in the two examples reported in Figure 7.

Finally, we wish to make some consideration about power consumption. The algorithm takes $41 \mathrm{~ms}$ for processing 104 samples acquired at $512 \mathrm{MHz}$ (which is approx. $20 \%$ of the CPU load), uses 25 KBytes ROM and 16 KBytes RAM. In these conditions, the wearable device in monitoring operation (i.e., saving data on the memory without transmitting via Bluetooth) dissipates $26 \mathrm{~mW}$. 
The device battery features $592 \mathrm{mWh}$, which means that the system has more than $20 \mathrm{~h}$ operation autonomy.
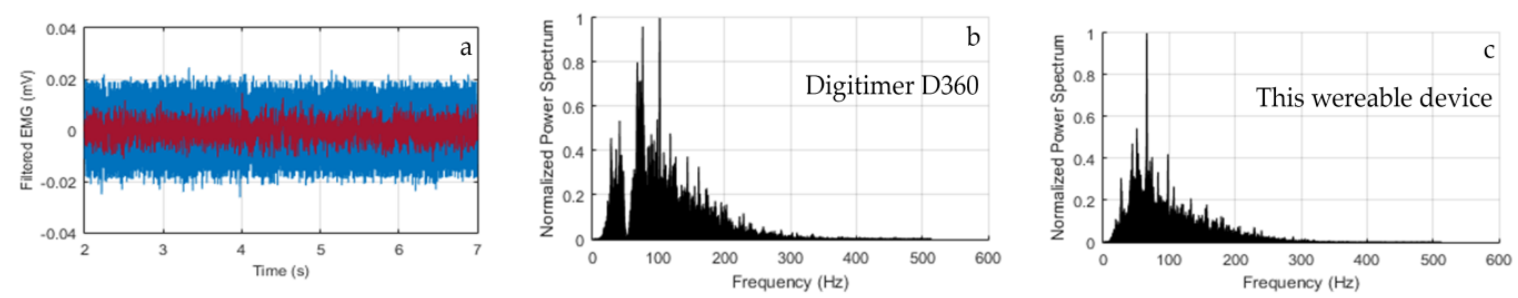

Figure 6. (a) Zoom of the baselines of the wearable device (red line) and the Digitimer D360 equipment (blue line); (b) FFT of the signals recorded with the Digitimer D360 and (c) our wearable device.
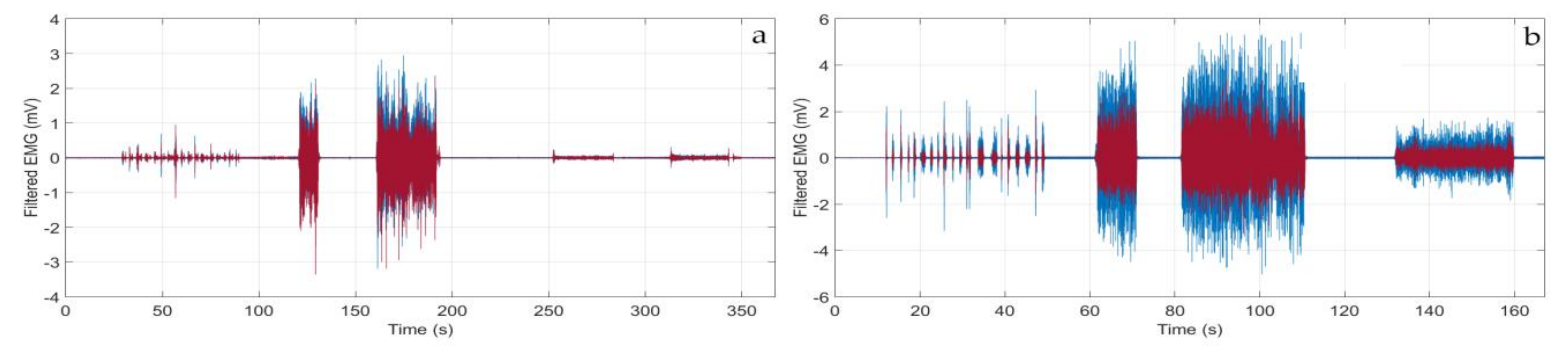

Figure 7. Filtered sEMG traces recorded with the wearable device (red line) and the Digitimer D360 equipment (blue line) in the case of: (a) brachial biceps contraction; (b) abductor pollicis brevis contraction.

In conclusion, the proposed wearable device is the first one embedding the sEMG data processing and performing continuous non-invasive monitoring of the muscle activity with high accuracy and long-time battery autonomy. It exhibits sensitivity and specificity in the detection of muscle activity comparable with those achieved with state-of-art wired equipment conventionally used in the hospitals, but with the advantage of being wireless and comfortably wearable.

Conflicts of Interest: The authors declare no conflict of interest.

\section{References}

1. Kita, A.; Lorenzi, P.; Rao, R.; Irrera, F. Reliable and Robust Detection of Freezing of Gait Episodes with Wearable Electronic Devices. IEEE Sens. J. 2017, 17, 1899-1908, doi:10.1109/JSENS.2017.2659780.

2. Lorenzi, P.; Kita, A.; Romano, G.; Rao, R.; Irrera, F. Mobile Devices for the Real Time Detection of Specific Human Motion Disorders. IEEE Sens. J. 2016, 16, 8220-8227, doi:10.1109/JSENS.2016.2530944.

3. Guyton, A.C. Textbook of medical physiology. J. Med. Educ. 1961, 36, 556.

4. Li, X.; Zhou, P.; Aruin, A.S. Teager-Kaiser Energy Operation of Surface EMG Improves Muscle Activity Onset Detection. Ann. Biomed. Eng. 2007, 35, 1532-1538.

5. Konrad, P. The ABC of EMG, A Practical Introduction to Kinesiology Electromyography, Version 1.4; Noraxon Inc.: Scottsdale, AZ, USA, 2006.

6. De Luca, C.J. The Use of Surface Electromyography in Biomechanics. J. Appl. Biomech. 1997, 13, 135-163.

(C) 2017 by the authors. Licensee MDPI, Basel, Switzerland. This article is an open access article distributed under the terms and conditions of the Creative Commons Attribution (CC BY) license (http://creativecommons.org/licenses/by/4.0/). 